\title{
Management of early period of hematopoietic cell transplantation in ambulatory/outpatient setting with primary antimicrobial prophylaxis: an attractive option
}

\author{
Jan Styczyński ${ }^{*}$ D, Agata Marjańska \\ Department of Pediatric Hematology and Oncology, Collegium Medicum in Bydgoszcz, Nicolaus Copernicus University in Toruń, \\ Jurasz University Hospital 1, Bydgoszcz, Poland
}

Both authors contributed equally to this study and share first co-authorship

\section{Abstract}

The concept of ambulatory/outpatient transplantations is based on variable distribution and location of performing basic stages of hematopoietic cell transplantation (HCT): central venous catheter insertion, high-dose chemotherapy administration, hematopoietic stem cells infusion, and supportive care during aplastic phase.

Our objective was to present the concept of outpatient transplantation and to review the available data on prophylaxis of infectious complications early after outpatient transplantation.

In our general recommendations, patients, family members and caregivers should be trained before discharge on the careful monitoring of fever and other signs and symptoms of infections. The clinical evaluation of patients should be performed twice weekly until clinical recovery. Standard transplant approach should be applied for dose of CD $34^{+}$cells, use of granulocyte colony-stimulating factor after HSC infusion, use of primary antimicrobial prophylaxis, and supportive care. Antimicrobial prophylaxis for ambulatory auto-HCT patients should be the same as for a conventional inpatient setting, including antibacterial, antiviral and anti-Pneumocystis jiroveci pneumonia (PjP) prophylaxis. For patients undergoing allo-HCT in an outpatient setting, the general recommendations are the same as for auto-HCT patients. Frequent monitoring of immunosuppressive treatment is recommended. Monitoring for cytomegalovirus infection and Aspergillus galactomannan should be the same as for conventional allo-HCT. Primary anti-mold prophylaxis is strongly suggested.

Ambulatory auto-HCT is feasible and safe, making this an important alternative option. This is an especially attractive option for multiple myeloma patients, as it has a very low transplant-related mortality risk. Obviously, ambulatory auto-HCT cannot be regarded as a routine procedure.

Key words: hematopoietic cell transplantation, outpatient transplantation, infectious complications, prophylaxis

Acta Haematologica Polonica 2021; 52, 3: 173-177

\section{Concept of outpatient setting}

The concept of ambulatory/outpatient transplantations is a consequence of variable distribution and location of performing four basic elements of hematopoietic cell transplantation (HCT) procedures: central venous catheter insertion, high-dose chemotherapy administration, hematopoietic stem cells infusion, and supportive care during aplastic phase [1]. Balancing between these four procedures results in differences between inpatient and

\footnotetext{
*Address for correspondence: Jan Styczyński, Department of Pediatric Hematology and Oncology, Collegium Medicum, Nicolaus Copernicus University Toruń, Skłodowskiej-Curie 9, 85-094 Bydgoszcz, Poland, phone +48 5258548 60, fax +48 5258540 87, e-mail: jstyczynski@cm.umk.pl Received: 15.03.2021 Accepted: 05.04.2021
} 
Table I. Models of outpatient transplantations (modified acc. to Martino et al. [3])

\begin{tabular}{|l|c|c|c|c|}
\hline Model & CVC insertion & HD therapy & HSC infusion & Aplastic phase \\
\hline Totally inpatient & Inpatient & Inpatient & Inpatient & Inpatient \\
\hline Early discharge & Inpatient & Inpatient & Inpatient & Outpatient \\
\hline Mixed & Outpatient & Outpatient & Inpatient & Outpatient \\
\hline Delayed admission & Outpatient & Outpatient & Outpatient & Inpatient \\
\hline Totally outpatient & Outpatient & Outpatient & Outpatient & Outpatient \\
\hline
\end{tabular}

$\mathrm{CVC}$ - central venous catheter; HD - high-dose; HSC - hematopoietic stem cell

outpatient settings (Table I) [2, 3]. Regardless of the inpatient/outpatient setting, the transplant itself carries the risk of typical transplant-related complications, namely toxicity and infections, and, in the case of allogeneic HCT, graft-versus-host disease (GvHD).

The objective of this paper was to present the concept of outpatient transplantation and to review the available data on infectious complications early after outpatient transplantation, with a focus on current recommendations for anti-infective prophylaxis in the early period after outpatient transplantation.

\section{Criteria for outpatient HCT}

An outpatient autologous HCT program was developed in 1993 with the aims of reducing hospitalization expenses and improving quality of life $[4,5]$. Performing HCT in an outpatient setting gives several advantages to the patient, including maintaining independence, decreasing the risk of nosocomial infections, better quality of life, and improved overall satisfaction. Outpatient HCT also provides advantages to the transplant center, including decreased use of resources, and reduced costs.

In order to perform HCT in an outpatient setting, cooperation between patient and transplant center is essential $[6,7]$. It is anticipated that patients qualified for this approach are usually expected not to have severe toxic or infectious complications. The main clinical indications for outpatient auto-HCT are multiple myeloma (after conditioning with melfalan) and lymphoma [both non-Hodgkin's lymphoma (NHL) and Hodgkin's lymphoma (HL, mainly after conditioning BEAM \{carmustine, etoposide, cytarabine, melphalan\}/BEAC \{carmustine, etoposide, cytosine arabinoside, cyclophosphamide\})], while rarely for other indications. Typical inclusion criteria include a high educational status and overall good clinical condition of the patient (Table II) $[2,3]$. It is important that the distance from the patient's house to the hospital can be covered within one hour.

As far as requirements for the transplant center are concerned, it is recommended that the hospital team should consist of at least two attending physicians, six advanced practice providers, six nurses, two dieticians, two clinical pharmacists, two schedulers, and a social worker.
Table II. Inclusion criteria for ambulatory/outpatient hematopoietic cell transplantation

\begin{tabular}{l} 
Patient \\
Patient having high educational status \\
Signed written informed consent \\
Age $\leq 65$ years \\
ECOG $\leq 2$ \\
Normal cardiac, lung, liver, and renal function \\
Absence of advanced disease \\
Status of primary disease: CR or PR \\
Absence of refractoriness to platelet transfusion \\
Absence of Gram-negative MDR pathogens colonization \\
Anti-infectious prophylaxis \\
Transplant center \\
\hline Outpatient clinic available $24 \mathrm{~h} /$ day or bed reserved \\
in Transplant Unit \\
Dedicated phone line $24 \mathrm{~h} /$ day \\
Availability of a caregiver $24 \mathrm{~h} /$ day \\
Detailed SOP for caregiver and patient \\
Home \\
\hline Distance from house to the hospital $\leq 1 \mathrm{~h}$ \\
Clean house
\end{tabular}

ECOG - Eastern Cooperative Oncology Group score; CR - complete remission; PR - partial remission; MDR - multi-drug resistant; SOP - standard operating procedure

If necessary, a patient staying at home should have immediate access to hospital facilities not only in an Outpatient Clinic, but also within night-time coverage by a physician available after hours in the system of inpatient setting $[8,9]$.

\section{Outpatient autologous HCT}

\section{Hospital readmission}

The three main indications for readmission include: persistent fever $>38^{\circ} \mathrm{C}$ without identified infectious source being the risk of severe sepsis with organ failure; severe oral mucositis or gastro-intestinal toxicity (grade III/IV) 
with insufficient liquid intake; and request of the patient resulting from psychological distress or loss of caregiver support. The risk of readmission is currently estimated to be $10-20 \%$ in patients with multiple myeloma [1], and $30-90 \%$ in patients with $\mathrm{NHL} / \mathrm{HL}[10,11]$, although as experience grows and clinical techniques improve, the risk of readmission has become lower in recent years. Recent data shows that in outpatient-HCT, with prophylaxis (such as ceftriaxone in patients with multiple myeloma, or piperacillin/tazobactam in cases of $\mathrm{NHL}$ and $\mathrm{HL}$ ), a relatively low rate of post-transplant febrile neutropenia can be achieved. This approach has resulted in good protection for patients at home, with an overall readmission rate of only $8.5 \%$ in a large cohort of 325 patients [2].

\section{Risk of early complications in outpatient versus inpatient auto-HCT groups}

In a meta-analysis of nine controlled or cohort studies including a total of 1,940 patients (no randomized clinical trial) [12] it was shown that in an outpatient setting the risk of developing febrile neutropenia was decreased by $56 \%$ [odds ratio $(\mathrm{OR})=0.44 ; p<0.0001]$, and the risk of developing septicemia was decreased by $60 \%$ (OR $=0.40 ; p=0.04)$. There was also a trend towards a lower risk of developing Clostridium difficile infection ( $\mathrm{OR}=0.73 ; p=0.4)$, a lower risk of developing mucositis grade $2-3(\mathrm{OR}=0.65 ; p=0.14)$, a lower risk of developing treatment-related mortality (TRM) $(\mathrm{OR}=0.37$; $p=0.12)$, and higher survival at 2 years $(O R=1.87 ; p=0.16)$. In all of these studies, granulocyte colony-stimulating factor (G-CSF) was used as a primary prophylaxis for patients in both inpatient and outpatient groups [12].

\section{Outpatient allogeneic HCT}

Data on outpatient allogeneic HCT is limited. However, a recently published large report from the United States included 1,037 patients at median age 58 years (range: 18-80) with hematologic malignancies, who received peripheral blood stem cells (PBSC) from $10 / 10$ or $9 / 10$ human leukocyte antigen (HLA)-matched related (MRD) or unrelated donor (MUD) between 1997 and 2017 [9]. These patients received non-myeloablative (NMA) or a reduced intensity conditioning $(\mathrm{RIC})$ regimen of $2 \mathrm{~Gy}(\mathrm{n}=862)$ or 3 Gy $(n=175)$ total body irradiation (TBI) with $(n=839)$ or without $(n=198)$ preceding fludarabine $30 \mathrm{mg} / \mathrm{m}^{2} /$ day on days $-4,-3$, and -2 . A small subset of patients received another regimen with $2 \mathrm{~Gy}$ TBI and clofarabine $30-50 \mathrm{mg} / \mathrm{m}^{2} /$ day $\times 5$ days $(n=34)$. Prophylaxis of GvHD after HCT included calcineurin inhibitor, 96 for MRD and 150-180 days for MUD: either cyclosporine $(n=433)$ or tacrolimus ( $n=164$ ), and sirolimus (180 days, $n=132$ ), or mycophenolate mofetil (28 days or 96 days, respectively).

Prophylaxis of infectious complications included: fluconazole for yeast prophylaxis, acyclovir for herpes simplex and varicella zoster virus prophylaxis, trimethoprim-sulfamethoxazole for Pneumocystis jirovecii prophylaxis (PJP). Patients were also monitored for cytomegalovirus (CMV) reactivation. All patients received prophylactic ursodiol from approximately 14 days before transplantation until at least day 180 after transplantation.

Cumulative incidence rate of hospital admission was $53 \%$, with a median 6 days of hospital stay. Two thirds of admissions occurred within first three weeks after HCT. The remaining $47 \%$ of patients were either never hospitalized or had only an overnight hospital stay for an infusion of PBSC. The main causes of hospitalization were neutropenic fever or infection (49\%), regimen-related toxicity (15\%), infusion of HCT (11\%), acute GvHD (8\%), cardiovascular complications $(7 \%)$, relapse $(2 \%)$, and others $(8 \%)$. Risk factors for hospitalization were: comorbidity index Hematopoietic Cell Transplantation - specific Comorbidity Index (HCT-Cl) value $2-3$ vs. $0-1$ (OR =1.6, $p=0.005)$ and $\geq 4$ vs. $0-1$ (OR $=2.4, p<0.0001)$, unrelated vs related donor $(\mathrm{OR}=2.4$, $p<0.0001)$, and HLA mismatch (OR =3.6, $p=0.006)$, while diagnosis of multiple myeloma was a protective factor $(\mathrm{OR}=0.6, p=0.003)$. Risk factors for TRM by day $100 \mathrm{in}$ cluded: age $\geq 50$ (OR =1.8, $p=0.003)$, unrelated vs related donor $(\mathrm{OR}=1.5, p=0.01)$, CMV (recipient $\mathrm{R}+$ or donor $\mathrm{D}+$ vs. $\mathrm{R}-/ \mathrm{D}-)(\mathrm{OR}=1.4, p=0.04)$, comorbidity index $\mathrm{HCT}-\mathrm{Cl}$ value $2-3$ vs. $0-1$ (OR =1.4, $p=0.05)$, and $\geq 4$ vs. $0-1$ (OR $=1.8, p=0.002$ ) [9].

In this cohort, 620 of the 1,037 patients died due to relapse $(n=342)$ or TRM $(n=278)$. Treatment-related mortality causes included GvHD (42\%); toxicity (14\%); age-related causes (such as cardiovascular, vascular, chronic obstructive pulmonary disease) (12\%); and infectious complications in the remaining 32\% (bacterial, fungal, or viral infections). Deaths from infectious complications occurred in 75 (84.3\%) patients who experienced GvHD: 30 acute GvHD grade 2/3, 11 chronic GvHD, and 34 both; while $14(15.7 \%)$ patients did not experience GvHD.

\section{Primary antimicrobial recommendations for outpatient transplants}

\section{General recommendations}

Patients, family members and caregivers should be trained before discharge on the careful monitoring of fever and other signs and symptoms of infections. The first clinical evaluation of a patient should be performed no later than day +5 after discharge, and then repeated twice weekly until sustained hematological engraftment and clinical recovery [3].

A standard transplant approach should be applied for dose of CD34 ${ }^{+}$cells, use of G-CSF after hematopoietic stem cell (HSC) infusion, use of primary antimicrobial prophylaxis and supportive care (hydration, management of nausea and vomiting, analgesic therapy, management of metabolic complications, and transfusion of blood products) $[2,13]$. 


\section{Auto-HCT}

Antimicrobial prophylaxis for ambulatory auto-HCT patients should be the same as for a conventional inpatient setting [14, 15], including antibacterial prophylaxis with quinolones, antiviral prophylaxis with acyclovir (at least up to three months after transplantation) and prophylaxis against Pneumocystis jiroveci with cotrimoxazole; primary antifungal prophylaxis is generally not recommended [3]. Real world data regarding infectious prophylaxis in outpatient auto-HCT studies has been summarized elsewhere [12].

\section{Allo-HCT}

For patients undergoing allo-HCT in an outpatient setting, the general recommendations are the same as for auto-HCT patients. The basic difference is the recommendation of frequent (2-3 times per week) monitoring of immunosuppressive treatment. Monitoring for CMV infection and Aspergillus galactomannan should the same as for conventional allo-HCT $[16,17]$. Primary anti-mold prophylaxis is strongly suggested $[2,9,18,19]$.

\section{Conclusions}

Ambulatory auto-HCT is feasible and safe, making it an important alternative. This is an especially attractive option for multiple myeloma patients, with a very low transplant-related mortality rate $[20,21]$. There is still concern (sometimes unjustified) about the lack of protective isolation during home stays that can predispose patients to a higher risk of infections. For multiple myeloma patients, auto-HCT in an outpatient setting is a safe and feasible option and should be considered by healthcare providers. Obviously, ambulatory auto-HCT cannot be regarded as a routine procedure. Nonetheless, many transplant centers are still hesitant about adopting this approach.

\section{Author contributions}

JS had primary responsibility for study design. Both authors performed analysis and wrote manuscript, contributed to data analysis and interpretation and critical revision of manuscript.

\section{Conflict of interest}

Both authors declared no conflict of interest related to this study.

\section{Financial support}

None.

\section{Ethics}

The work described in this article has been carried out in accordance with The Code of Ethics of the World Medical Association (Declaration of Helsinki) for experiments involving humans; EU Directive 2010/63/EU for animal experiments; Uniform requirements for manuscripts submitted to biomedical journals.

\section{Acknowledgements}

This paper is based on the lecture "Management of early complications in an ambulatory/outpatient setting: early infectious complications' presented by the first author during the Virtual EBMT Annual Meeting on 14-17 March 2021.

\section{References}

1. Martino M, Lemoli RM, Girmenia C, et al. Italian consensus conference for the outpatient autologous stem cell transplantation management in multiple myeloma. Bone Marrow Transplant. 2016; 51(8): 1032 -1040, doi: 10.1038/bmt.2016.79, indexed in Pubmed: 27042841.

2. Fernandez-Aviles F, Gutierrez-Garcia G. At-Home HSCT. In: Carreras E, Dufour C, Mohty M, Kroger N. ed. The EBMT handbook: hematopoietic stem cell transplantation and cellular therapies. Springer, Cham 2019: 467-471.

3. Martino M, Paviglianiti A, Memoli M, et al. Multiple myeloma outpatient transplant program in the era of novel agents: state-of-the-art. Front Oncol. 2020; 10: 592487, doi: 10.3389/fonc.2020.592487, indexed in Pubmed: 33262948.

4. Peters WP, Ross M, Vredenburgh JJ, et al. The use of intensive clinic support to permit outpatient autologous bone marrow transplantation for breast cancer. Semin Oncol. 1994; 21(4 Suppl 7): 25-31, indexed in Pubmed: 7916487.

5. Jagannath S, Vesole DH, Zhang M, et al. Feasibility and cost-effectiveness of outpatient autotransplants in multiple myeloma. Bone Marrow Transplant. 1997; 20(6): 445-450, doi: 10.1038/sj.bmt.1700900, indexed in Pubmed: 9313876.

6. Dytfeld D, Łojko-Dankowska A, Nowicki A, et al. Safety and cost effectiveness of outpatient autologous hematopoietic stem cell transplantation for multiple myeloma - single center experience of a pilot Early Discharge Program. Acta Haematol Pol. 2021; 52(3): 178-181, doi: 10.5603/AHP.a2021.0029.

7. Jakubowiak A. Outpatient transplants: American standard in Poland. Acta Heamatol Pol. 2021; 52(3): 145-146, doi: 10.5603/ /AHP.2021.0039.

8. Shah NA. Allogeneic hematopoietic cell transplantation in the outpatient setting. Biol Blood Marrow Transplant. 2019; 25(11): e319-e320, doi: 10.1016/j.bbmt.2019.09.031, indexed in Pubmed: 31585166.

9. Granot N, Storer BE, Cooper JP, et al. Allogeneic hematopoietic cell transplantation in the outpatient setting. Biol Blood Marrow Transplant. 2019; 25(11): 2152-2159, doi: 10.1016/j.bbmt.2019.06.025, indexed in Pubmed: 31255743.

10. Faucher C, Le Corroller Soriano AG, Esterni B, et al. Randomized study of early hospital discharge following autologous blood SCT: medical outcomes and hospital costs. Bone Marrow Transplant. 2012; 47(4): 549-555, doi: 10.1038/bmt.2011.126, indexed in Pubmed: 21725375 .

11. Scortechini I, Montanari M, Mancini G, et al. Conditioning regimen with BCNU, etoposide, cytarabine and melphalan plus amifostine for outpatient autologous stem cell transplant: feasibility and outcome in 97 patients with lymphoma. Leuk Lymphoma. 2014; 55(7): 1657 1660, doi: 10.3109/10428194.2013.842989, indexed in Pubmed: 24024474. 
12. Owattanapanich W, Suphadirekkul K, Kunacheewa C, et al. Risk of febrile neutropenia among patients with multiple myeloma or lymphoma who undergo inpatient versus outpatient autologous stem cell transplantation: a systematic review and meta-analysis. BMC Cancer. 2018; 18(1): 1126, doi: 10.1186/s12885-018-5054-6, indexed in Pubmed: 30445930.

13. Styczyński J. Infectious complications in children and adults with hematological malignancies. Acta Haematol Pol. 2019; 50(3): 167-173, doi: 10.2478/ahp-2019-0027.

14. Styczyński J, Czyżewski K, Ussowicz M, et al. Antimicrobial prophylaxis in patients after hematopoietic cell transplantation: results of a survey of the Polish Federation of Bone Marrow Transplant Centers. Acta Haematol Pol. 2020; 51(3): 183-186, doi: 10.2478/ahp-2020-0032.

15. Bartoszewicz N, Czyżewski K, Dębski R, et al. Efficacy of keratinocyte growth factor in prevention of oral mucositis in children undergoing allogeneic hematopoietic cell transplantation. Acta Haematol Pol. 2020; 51(3): 172-178, doi: 10.2478/ahp-2020-0030.

16. Styczyński J. ABC of viral infections in hematology: focus on herpesviruses. Acta Haematol Pol. 2019; 50(3): 159-166, doi: 10.2478/ /ahp-2019-0026.
17. Styczyński J. Prophylaxis vs preemptive therapy in prevention of CMV infection: new insight on prophylactic strategy after allogeneic hematopoietic cell transplantation. Acta Haematol Pol. 2020; 51(1): 17-23, doi: 10.2478/ahp-2020-0005.

18. Gil L, Kałwak K, Piekarska A, et al. Antifungal management in adults and children with hematological malignancies or undergoing hematopoietic cell transplantation: recommendations of Polish Society of Hematology and Blood Transfusion, Polish Society of Pediatric Oncology and Hematology, and Polish Adult Leukemia Study Group, 2020. Acta Haematol Pol. 2020; 51(2): 60-72, doi: 10.2478/ahp-2020-0014.

19. Styczyński J, Czyżewski K, Frączkiewicz J, et al. Clinical spectrum and outcome of invasive mucormycosis in children and adults: Polish experience of the decade 2010-2019. Acta Haematol Pol. 2020; 51(3): 157-163, doi: 10.2478/ahp-2020-0028.

20. Jurczyszyn A, Hutch R, Waszczuk-Gajda A, et al. Monoclonal gammopathies of undetermined significance and smoldering myeloma. Acta Haematol Pol. 2020; 51(4): 193-202, doi: 10.2478/ahp-2020-0035.

21. Giebel S, Basak G, Bieniaszewska M, et al. Current status and achievements of Polish haemato-oncology. Acta Haematol Pol. 2021; 52(1): 4-17, doi: 10.5603/AHP.2021.0003. 